Para enlazar con este artículo / To link to this article:

http://dx.doi.org/10.6035/MonTI.2019.ne5.2

Para citar este artículo / To cite this article:

Fernández Souto, Ana Belén; José Rúas Araújo \& Adriana Prada Iglesias. (2019) "Más allá de la proximidad cultural: vínculos entre el idioma gallego y el portugués en la negociación internacional." En: Montero Küpper, Silvia; Montse Vázquez Gestal \& Iván Puentes Rivera (eds.) 2019. Comunicación, Traducción e Interpretación / Communication, Translation and Interpreting. MonTI Special Issue 5, pp. 45-71.

\title{
MÁS ALLÁ DE LA PROXIMIDAD CULTURAL: VÍNCULOS ENTRE EL IDIOMA GALLEGO Y EL PORTUGUÉS EN LA NEGOCIACIÓN INTERNACIONAL ${ }^{1}$
}

\author{
BEYOND THE LINGUISTIC PROXIMITY: THE GALICIAN AND \\ THE PORTUGUESE IN THE INTERNATIONAL NEGOTIATION
}

\author{
ANA BELÉN FERNÁNDEZ SOUTO \\ abfsouto@uvigo.es \\ Universidade de Vigo \\ JOSÉ RÚAS ARAÚJO \\ joseruas@uvigo.es \\ Universidade de Vigo \\ AdRIANA PRADA IGLESIAS \\ Universidade de Vigo
}

\section{Resumen}

Planteamos una reflexión sobre el uso del idioma gallego como lengua vehicular entre el portugués y el español. A partir de varias acciones institucionales, proponemos un estudio comparativo sobre el uso de la lengua gallega en los negocios en el ámbito de

1. Agradecimientos: Este trabajo fue realizado gracias a la colaboración del personal de las embajadas de España en Brasil, Portugal, Timor Oriental, Cabo Verde y Angola. También destacamos la ayuda de la Rede Pexga (Xunta de Galicia), así como de la Confederación de Empresarios de Galicia en Brasil. 
la lusofonía, tomando como referencia varias campañas promocionales del gobierno de la Xunta de Galicia. La investigación recopila datos mediante entrevistas en profundidad a responsables de las embajadas de cinco países lusófonos y a empresarios gallegos que negocian en los mismos. La hipótesis manejada es que la utilización del gallego contribuye al entendimiento dentro del ámbito de la lusofonía, pero hace falta una mayor inmersión en el idioma a la hora de afrontar una negociación internacional; este proceso de inmersión lingüística también debe incluir conocimientos culturales como el uso del protocolo y el ceremonial, el valor de la gastronomía o el sentido del tiempo, entre otros.

Palabras clave: Comunicación; Idioma; Gallego; Portugués; Empresarios

\section{Abstract}

This is a reflection about the use of the Galician language as a language between the Portuguese and the Spanish.

Based on several institutional actions, we propose a comparative study on the use of the Galician language in business abroad in countries of lusophony, taking as a reference different promotional campaigns of the government of the Xunta de Galicia.

The research will work with the data of several interviews with embassy officials from five Portuguese-speaking countries and Galician businessmen who negotiate there.

Keywords: Communication; Language; Galician; Portuguese; Business

\section{Introducción}

Galicia y Portugal guardan importantes vínculos históricos, culturales, sociales y económicos entre sí. El idioma es el más evidente, ya que ambas lenguas tienen un origen histórico común: el galaico-portugués; lenguas que constituyen un mismo sistema lingüístico y que debería considerarse (Ledo-Andión: 2011), por sí mismo, una pieza de inestimable valor de uso -y de intercambio- para la circulación de objetos y sujetos de comunicación.

Este artículo pretende centrarse en el ámbito sociolingüístico desde el punto de vista de la comunicación empresarial y el protocolo internacional, e intenta también hacer extensiva la investigación a otros países de habla lusófona con la intención de verificar el grado de proximidad cultural existente entre Galicia y esos países. 
Tomamos como punto de partida ese nexo histórico y social que une ambas culturas y, sobre todo, el consenso entre los diversos actores sociolingüísticos gallegos sobre la conveniencia de explotar el potencial del idioma en las relaciones con los países de habla lusófona.

Sin embargo, a pesar de esta proximidad, a la hora de entablar una relación internacional se hace necesario evitar ruidos comunicativos que, en muchos casos, van más allá del ámbito propiamente lingüístico y vienen dados por malentendidos que proceden de la comunicación no verbal o de la falta de conocimiento de otros de factores de carácter cultural que construyen la identidad de un país.

Para llegar a entender la particular situación del uso de la lengua gallega en el ámbito lusófono, presentamos un estudio en el que se parte de la conceptualización de la comunicación internacional en el ámbito de los negocios, con el propósito de concretar las diferencias socioculturales identificadas desde las distintas embajadas de España en los países de habla lusófona, así como a través de la experiencia descrita por distintos empresarios gallegos que operan en estos países. Diferencias que pueden llegar a convertirse en ruidos comunicativos y entorpecer una relación bilateral, por lo que es necesario conocerlas para poder salvarlas.

\section{Objetivos y supuesto de partida}

Los objetivos de esta investigación son los siguientes:

1. Plantear una reflexión teórica sobre el papel que juega el factor cultural en los negocios internacionales y cómo el idioma es uno de los elementos más importantes para el establecimiento de lazos de unión en una sociedad.

2. Estudiar el fenómeno de la globalización con la pretensión de dibujar un marco teórico que defina las repercusiones de ese fenómeno en la economía mundial.

3. Investigar sobre la lusofonía y analizar su espacio cultural con el objetivo de poder rescatar información útil con la que ayudar al tejido empresarial e institucional gallego que busca negociar en ese entorno. 
4. Comprender el potencial del gallego como lengua de negocios en el ámbito lusófono, partiendo de que entre gallego y portugués existen grandes similitudes.

5. Llevar a cabo una comparativa sobre los datos del espacio cultural lusófono a partir de una muestra de cinco países en la que se recojan los principales aspectos de la comunicación verbal y no verbal que hay que tener en cuenta en la negociación internacional.

El supuesto de partida es: la cercanía lingüística entre el gallego y el portugués es evidente y va más allá de la mera concepción idiomática, llegando a ámbitos como el sociocultural, político y económico, especialmente en el caso de Portugal, dada su proximidad. Sin embargo, esta cercanía no es total y se acentúa al comparar el contexto gallego con el de otras regiones lusófonas, donde se evidencian limitaciones para el entendimiento y ruidos comunicativos.

\section{Metodología}

Utilizaremos una metodología fundamentalmente deductiva, a partir de supuestos teóricos y prácticos. En un primer momento se elabora un marco teórico basado en diversas fuentes bibliográficas para, a partir de ahí, llegar al estudio concreto de la cultura lusófona y gallega con la intención de analizar sus principales puntos de encuentro y aquellos que pueden convertirse en ruidos comunicativos.

A continuación, se plantea una entrevista en profundidad para recabar datos sobre cuestiones culturales, aspectos relacionados con el protocolo, la cronémica, proxémica, kinesia y el modelo de negociación puesto en práctica. Esta entrevista se realizó al personal funcionario de las embajadas y/o consulados de España en distintos países de habla portuguesa, en concreto, Angola, Cabo Verde, Brasil, Portugal y Timor Oriental.

Con respecto de la selección de los países que deben ser estudiados, pretendemos abarcar un país por continente, escogiendo aquel con mayor número de hablantes, y de ahí que se analice Portugal, por la parte europea; Brasil, por la americana; Timor Oriental, de Asia; y los casos de Cabo Verde y Angola como representación del continente africano. Dado el crisol cultural existente en el continente africano, se han seleccionado estos dos países para su estudio. 
El personal entrevistado en el caso de las embajadas ha sido el indicado desde las propias instituciones, con las que se contactó para solicitar una entrevista con la persona cuyo perfil mejor encajase con las necesidades de la investigación.

Esta entrevista también fue realizada a varios empresarios gallegos afincados en esos países con experiencia en el trato internacional y en el uso de la lengua gallega en la negociación con la cultura lusófona. Su selección y contacto ha venido facilitado por la Rede Pexga, dependiente del Instituto Galego de Promoción Exterior, (IGAPE).

Sobre el contenido de la entrevista, cabe destacar que se planificó en seis bloques específicos:

- Bloque 1: La sociedad y los negocios, donde se busca información sobre los rasgos generales de tradiciones y ritos, el papel de la mujer, el sentido del tiempo, la comida y bebida o el transporte.

- Bloque 2: Las relaciones con otras personas, en la que se recopilarán datos sobre las presentaciones, los saludos y tratamientos, los títulos profesionales y la importancia de la jerarquía.

- Bloque 3: El lenguaje no verbal. Analiza la proxemia y la kinesia.

- Bloque 4: El lenguaje verbal, que se centra en el estudio de la paralingüística.

- Bloque 5: La negociación, que incluye el análisis sobre las estrategias de negociación, el papel del negociador extranjero y la propuesta de trabajo, el uso de las tarjetas profesionales, los regalos, las invitaciones, los espacios donde se desarrolla la negociación y otros aspectos a tener en cuenta en el transcurso de la negociación.

- Bloque 6: De carácter genérico, en el que el entrevistado puede hacer referencia a cualquier otro aspecto.

Las entrevistas se plantearon a través de un cuestionario de preguntas cerradas y se realizaron por vía telefónica o por videoconferencia, dependiendo de las necesidades del entrevistado.

A partir de todos estos datos, una vez analizados y comparados con el caso gallego, establecemos unas conclusiones que recogen el papel actual de ambas lenguas, gallego y portugués respecto de su uso común en el ámbito 
de la negociación internacional, así como la importancia que tiene en esa interacción el conocimiento mutuo respecto a las diferencias culturales.

\section{Globalización económica y negociación internacional}

La globalización económica condiciona los mercados internacionales y las relaciones bilaterales entre países y regiones.

Otero (2008) asegura que la globalización es un proceso en el que la actividad económica de un país traspasa las fronteras y busca integrar a los clientes y productos de otros países del mundo y donde los límites nacionales de la actividad económica se van difuminando para facilitar un acceso más libre a los factores productivos y a los mercados de bienes.

Muy vinculado a este término está el de "negocio internacional", que Daniels, Radebaugh \& Sullivan (2004) entienden como "toda transacción comercial, privada el gubernamental entre de los o más países". Lo que caracteriza este tipo de transacciones no es el hecho de que se lleven a cabo entre dos o más países, ni tampoco que sean de carácter comercial o personal, sino que al producirse implican la necesidad de comunicarse con personas de una cultura diferente a la nuestra.

Además, hoy en día, el denominado "capital intelectual y relacional" forma parte indisoluble de la cultura empresarial a través de una serie de activos intangibles que contribuyen a la creación de valor añadido, hasta el punto de que las mejores empresas son las que buscan activos de conocimiento mejor y más rápido que la competencia, un aspecto ya estudiado en el sector de la automoción de Galicia y el Norte de Portugal (Da Silva, Froilán y Fernández, 2009), considerado como factor esencial para la innovación.

\subsection{Breve nota sobre las tendencias que configuran la economía global actual}

La economía mundial de hoy lleva implícitas una serie de tendencias que corroboran la necesidad de las empresas de internacionalizarse y de realizar negocios en el contexto global. Tal como apoya Carbaugh (2009: 53) "todos los aspectos de la economía de una nación (sus industrias, niveles de ingreso y empleo, así como estándares de vida) se vinculan con las economías de sus socios comerciales". 
Las tendencias socioeconómicas que rigen las relaciones internacionales del presente siglo son (González Gurriarán et al. 2000):

- El crecimiento progresivo del comercio exterior.

- El aumento continuado de la producción mundial.

- La progresión incesante de la inversión directa en el exterior.

- El desarrollo constante de la transferencia internacional de tecnología.

- El incremento progresivo de los movimientos internacionales de capitales.

- La creciente significación económica de la cooperación internacional.

- El aumento de la regionalización de la economía mundial.

\subsection{Sobre la cultura, la comunicación y negociación internacional}

Como señala Maldonado (2006), la cultura es el elemento marginal de las negociaciones internacionales que solamente se tiene en cuenta cuando se produce el bis a bis con nuestro interlocutor extranjero y surgen los primeros malentendidos, ocasionados por el inevitable contraste cultural que se produce entre ambas partes.

Para evitar esas interferencias culturales y hacer que cualquier cuestión se resuelva de manera fluida y ágil, el primer paso es conocer en profundidad la cultura de la otra parte y conferirle el peso que realmente tiene en el campo de las relaciones internacionales.

Es importante conocer los elementos que componen una cultura, aunque a veces resulta difícil enumerarlos, dado que la concepción de unos autores y otros varía considerablemente (Rodríguez 2007). En cualquier caso, se considera que una cultura se compone de los siguientes elementos (Rugman \& Hodgetts 1997):

- La religión.

- Los valores y actitudes.

- Los modales y las costumbres.

- La estética.

- La educación.

- Los elementos materiales.

- La organización social. 


\subsection{Un elemento cultural especial: el idioma (verbal y no verbal)}

El idioma es el principal medio de comunicación que emplean los individuos de una sociedad para comunicarse. Cuando pensamos en ese concepto, la mente nos traslada al universo de las palabras y de la comunicación verbal. Sin embargo, el idioma también se refleja en los gestos y en la expresión corporal de las personas; es lo que se conoce como "comunicación no verbal".

Cuando un empresario pretende negociar en el ámbito internacional, debe demostrar ciertas competencias en el idioma en que se comunica con el interlocutor extranjero porque solamente así podrá entablar un diálogo verdaderamente eficaz, entender todos los matices de su cultura, descodificar la información sin necesidad de un intérprete y descifrar los dobles sentidos de ciertas frases y expresiones que adquieren un significado determinado dado el contexto en el cual nos encontremos.

El lenguaje no verbal, según Cuadrado (2007), engloba todos sus aspectos más característicos en relación a tres conceptos clave: la kinesia, la paralingüística y la proxemia. A este listado inicial, añadiremos una variable más: la cronémica.

La kinesia estudia los mensajes que transmite nuestro cuerpo a través de los movimientos que realiza, las expresiones que manifiesta e incluso el aspecto físico que muestra. La paralingüística, por su parte, analiza los matices que suenen acompañar la manera de hablar, tales como el tono de voz, el ritmo, las pausas y la velocidad de pronunciación. La proxemia descifra los mensajes que se extraen del espacio en el cual nos movemos y del uso que hacemos del espacio personal. Por último, la cronémica es la variable que hace referencia al uso del tiempo.

Las dimensiones del idioma traspasan la frontera oral y se dejan entrever en otros aspectos de la vida que deben ser tenidos en cuenta por cualquier interlocutor, sea empresario o no, con ganas de abrirse camino en el exterior estableciendo una relación interpersonal adecuada a los cánones culturales.

Según Watzlawick (1971) parece imposible no comunicar, ya que cada gesto, cada acción, cada práctica sociocultural es significada por otros, e incluso es entendida como intencional, de ahí la necesidad de ser consciente de las diferencias culturales y de los posibles ruidos comunicativos o interferencias comunicativas posibles al entrar en contacto varias culturas diferentes. 


\subsection{Requisitos para una efectiva comunicación intercultural}

Como requisitos para la comprensión mutua en el ámbito intercultural destacaremos:

a. Conocer la cultura propia. Cuando alguien es consciente de sus bases culturales, éstas dejan de ser un obstáculo para la comunicación eficaz. Esto permite tener sensibilidad de cara a otras identidades. Es necesario documentarse sobre las posibles diferencias y hacerlo utilizando fuentes fiables, como embajadas, consulados o, en su caso, asociaciones de empresarios y profesionales que conozcan ambas culturas y puedan actuar de intermediarios.

b. Es importante la aceptación del principio de la relatividad cultural, que indica que no existen culturas superiores o inferiores a las otras.

c. Las primeras impresiones perduran.

d. Eliminar las posibles interferencias de la comunicación, tanto desde el punto de vista lingüístico como desde la comunicación no verbal.

e. Informarse de antemano sobre los interlocutores, tanto a título individual como colectivo.

f. Dominar las técnicas de negociación, ya que existen culturas más cálidas o frías, más o menos temperamentales, con mayor o menor valoración del sentido del tiempo.

g. Es necesario saber recibir y ser recibido, demostrando una rápida capacidad de actuación y de adaptación constante.

\section{El gallego y la negociación internacional}

Los negocios son cada vez más internacionales, por lo que se hace imprescindible disponer de una inteligencia social en el marco de la negociación intercultural. Galicia no escapa de esta tendencia y su necesidad de internacionalización parece mayor que la del resto de España, según afirma López Facal (2012: 20), ya que las mercancías objeto de intercambio son las propias de una economía industrial moderna y la comunidad gallega ocupa el sexto puesto en el ránking nacional español de exportaciones, según el Instituto Gallego de Promoción Empresarial (Igape). Con la entrada en la UE, la economía gallega sufrió un "choque brutal", como categoriza Sáez Ponte (2012), por la pérdida de competitividad de alguno de sus principales sectores productivos, pero esta 
situación aproximó el tejido empresarial gallego al portugués, especialmente con la creación de la Eurorrexión, que trajo importantes ayudas que sirvieron para modernizar las infraestructuras, fundamentalmente.

Esto provocó que durante el período comprendido entre 1993 y el año 2000, Galicia empezase a exportar, en primer lugar, a Portugal, para luego sacar partido del resto del mercado europeo. Como consecuencia, en la pasada década el PIB gallego creció más que el español y se caracterizó por tener un balance comercial favorable (Sáez Ponte 2012: 18).

Pese a todo, el tejido empresarial gallego necesita diversificar más los mercados exteriores para minimizar los impactos negativos de una excesiva concentración de riesgo en el mercado interior europeo. Cordal (2012: 22) refuta la idea al afirmar que "nuestra acción exterior no puede ceñirse al marco europeo (...) y debemos ser ambiciosos para conseguir otras metas".

En consecuencia, los empresarios gallegos se ven en la necesidad de acudir a mercados emergentes y tanto su ubicación geográfica como los factores culturales y lingüísticos posicionan especialmente a Galicia para entablar relaciones con países consolidados y emergentes en el escenario económico mundial, como Brasil o Angola.

Además, la proximidad geográfica de Galicia con Portugal facilitó, durante la última década, el intercambio, con la apertura de nuevas vías para la movilidad, formación y empleo en el ámbito transfronterizo (Rial, Valcarce, Barreira et al. 2012), a través de la constitución de la eurorregión Galicia-Norte de Portugal y el proyecto Eixo Atlántico, integrado por distintas ciudades gallegas y del norte de Portugal. Un hecho que contribuyó al fortalecimiento de las relaciones a nivel económico, político e institucional en dicho espacio (Trillo \& Lois 2011).

\subsection{La gran ventaja del idioma}

Cada país utiliza un código lingüístico propio, pero son muchos los que a pesar de tener valores, tradiciones y concepciones diferentes de la sociedad emplean la misma lengua. Basándose en esto surgen espacios geolingüísticos (Melo Alves 2007) organizados como fuertes instrumentos de promoción y cooperación dentro de la globalización de las relaciones internacionales. 
Los países que forman estos espacios saben que su fuerza reside en la unión, y que esa unión reside en su nexo común: la lengua. Cuando la lengua, además de valor comunicativo y cultural, adquiere una vertiente estratégica, puede suponer un instrumento poderoso en otros campos que no abarcan cuestiones propiamente lingüísticas. Así, el poder económico de una lengua reside en el número de hablantes con los que cuenta y en las estadísticas financieras de todos los países que se identifican con ella.

En este sentido, la lengua gallega, en referencia al portugués, cuenta con un gran potencial, según los supuestos marcados por Antero et al. (2012):

- Los que hablan la misma lengua, o una semejante, tienen más posibilidad de establecer relaciones entre ellos.

- Los que conocen la lengua de otro están más abiertos a entender su cultura y sus valores.

- Cuanto mayor es el número de hablantes de una lengua, más valor adquiere a nivel global. Por lo tanto, más personas se encuentran dispuestas a aprender esa lengua y otras tantas se apresuran a hablarla.

- El uso de la misma lengua permite más facilidad de comunicación entre entidades o individuos dispares.

- El dominio de varias lenguas es hoy en día un factor diferenciador.

- El sentimiento de pertenencia.

Por lo tanto, es lógico que el espacio lusófono interese a los empresarios gallegos, así como otros profesionales de la comunidad, que ven en él una gran oportunidad.

\subsection{El gallego en el ámbito de la lusofonía}

Galicia guarda un estrecho vínculo histórico con Portugal y su lengua (Bagno 2011: 36), hasta el punto de existir ciertas disputas en relación a su parentesco (Monteagudo \& Pintos 2010: 80).

Dejando a un lado este conflicto (para los que rechazan el vínculo con Portugal y priman el lazo a España o aquellos otros que rechazan el vínculo a España y priman el portugués), es importante identificar el término "lusofonía", que viene delimitado por el conjunto de países que tienen como lengua oficial el portugués. 
Respecto al gallego, Monteagudo \& Pintos (2010), consideran que la relación con la lusofonía puede ser posible y viable si las relaciones entre el gallego y el portugués se vuelven más activas, dejan de ser solamente teóricas e implican a un mayor número de personas. Coincide también Castro (2009: 249), quien entiende que Galicia es un enclave estratégico para establecer relaciones con los mercados europeos, los latinos y africanos, así como un pueblo con facilidad para manejarse en el espacio lusófono.

¿Cuáles son los vínculos entre ambas culturas que pueden ser ventajas competitivas en el marco de la lusofonía? Según Álvarez (2009: 259), destacan las siguientes:

- La afinidad lingüística.

- La historia y el pasado común.

- La vecindad geográfica entre Galicia y Portugal.

- La posición atlántica estratégica de Galicia y Portugal, que permite a la Unión Europea establecer negocios de índole internacional con América y África.

- Las semejanzas culturales y de hábitat.

- La estrecha relación en ámbitos como el científico y el cultural.

- El peso de la emigración del pueblo gallego hacia países de habla portuguesa.

- La pertenencia al mapa pescador.

\subsection{La promoción del idioma gallego}

La Constitución de 1978 española permitió el establecimiento de lenguas oficiales diferentes del español en algunos territorios del Estado y es a partir de entonces cuando se empiezan a hacer públicas las líneas de promoción lingüística desarrolladas desde distintos entes institucionales, en un primer lugar, promocionando el uso interno de la lengua y, años más tarde, promoviendo su uso fuera de Galicia.

Esta segunda línea de actuación se hizo más evidente en los últimos años, con diversas reivindicaciones del valor de mercado de la lengua como instrumento comunicativo para relacionarse con los países lusófonos. Sirvan como ejemplo las declaraciones del expresidente del gobierno autonómico, Xerardo Fernández-Albor, en O Xornal de Galicia del 23 de mayo de 2010, 
refiriéndose a la importancia del gallego como vehículo para aprender portugués: "Tenemos una opción fantástica para relacionarnos con Brasil y con los países lusófonos de África".

En la misma dirección apuntan las palabras del Director Xeral de Política Lingüística de la Xunta de Galicia, Valentín García, en el periódico Galicia Confidencial (17 de mayo de.2013): "A lusofonía é unha oportunidade para o galego. Nós enfocámola, ademais, cara ao sector económico porque [...] os nosos empresarios, a diferencia dos cataláns, nunca se destacaron pola defensa do idioma, aínda que isto está mellorando. Por iso vimos a oportunidade de explicarlle a estes empresarios a importancia que teñen co idioma para exportar a países lusófonos. Para nós o contacto coa lusofonía é moi importante."

El Director de Proyectos de la Federación Galega de Parques Empresariales de Galicia, Iván Vaqueiro (2013), afirma también que "É certo que o galego é uma oportunidade, mas se calhar precisaríamos de melhorar as ferramentas de comunicação desde uma ótica setorial, técnica e específica da idiossincrasia desses países. O galego complementa-se com o português e vice-versa, e como tudo numa atividade empresarial, se se fizer, há de se fazer bem, e para isso a Junta deve mobilizar fundos para ações formativas neste âmbito."

Redundando en esta idea, destacaremos algunas acciones institucionales de promoción del gallego en el entorno empresarial y, sobre todo, en el ámbito de la negociación internacional:

- "O galego, chave para os teus negocios no mundo": Es una campaña conjunta que lanzó la FEGAPE (Federación Galega de Parques Empresariales) y la Secretaría Xeral de Política Lingüística de la Xunta de Galicia en el año 2012. Pretendía incentivar al empresariado gallego para que utilizara su propio idioma a la hora de hacer negocios. La campaña se llevó a cabo mediante en formato impreso con distribución en la red empresarial asociada al FEGAPE y los organismos económicos más destacables de la Comunidad.

2. http://www.lingua.gal/o-galego/promovelo/rede-de-dinamizacion-linguistica/ programas/_contido_0103/galego-chave-para-teus-negocios-mundo 
- "Exportar en galego3": En el año 2013 se lanzó una nueva campaña que daba continuidad a la iniciativa anterior. De nuevo de la mano de la Secretaría Xeral de Política Lingüística y FEGAPE se presentó una guía práctica para los empresarios interesados en conocer mejor las posibilidades del mercado lusófono.

- "ConsumoGustos"": Iniciativa del Foro Enrique Peinador y de la Asociación Galega de Adegueiros, en la que colaboraba la Consellería de Cultura, Educación e Ordenación Universitaria, a través de la Secretaría Xeral de Política Lingüística. Consumogustos comenzó en 2012 y promovía el etiquetado en gallego de los productos alimentarios cultivados en Galicia.

- Como colofón a todas estas acciones de promoción debemos destacar que en 2016 el Gobierno de la Xunta de Galicia presentó el "Plan de dinamización da lingua galega no tecido económico", que pretendía materializarse entre los años 2016 y 2020 implicando a los principales agentes administrativos y empresariales de Galicia. Trataba de poner en marcha un abanico de posibilidades para reforzar y aumentar la presencia del gallego en la economía. El plan se estructuraba a través de tres objetivos: a) Crear conciencia de que el uso del gallego favorece los resultados y objetivos empresariales y las relaciones laborales, comerciales y bancarias; b) Extender el uso de la lengua gallega en el ámbito económico, en las relaciones laborales, comerciales y empresariales y c) Utilizar el idioma para impulsar una imagen de marca diferencial propia de Galicia y sus productos vinculados a la calidad y la innovación.

Sin embargo, la cercanía estructural que mantienen gallego y portugués tampoco puede llamar a nadie a engaño: la comunicación efectiva entre hablantes de ambas lenguas no está exenta de obstáculos.

3. http://www.lingua.gal/o-galego/promovelo/rede-de-dinamizacion-linguistica/ programas? content=/Portal-Lingua/Contido/iniciativa/Programas/contido_0101.html

4. http://www.lingua.gal/c/portal/layout?p_l_id=1639655\&content=Programas/ contido_0103.html

5. http://www.edu.xunta.gal/portal/es/node/19831 


\section{Estudio comparado de los usos protocolarios y sociales del gallego frente a los del ámbito de la lusofonía}

Para abordar este epígrafe recopilamos la información a través de entrevistas realizadas a empresarios gallegos y trabajadores de las embajadas españolas en Portugal, Angola, Cabo Verde, Brasil y Timor Oriental.

\subsection{Brasil}

Brasil cuenta con una población que se caracteriza por un espíritu emprendedor y alegre, también está marcado por cierto carácter despreocupado y bajos niveles de eficiencia. La religión está muy presente. Es una sociedad de contacto. Resultan muy expresivos en el ámbito verbal y no verbal.

La sociedad brasileña es muy diversa y una de sus principales características es la mezcla cultural, lo que se hace notar en la convivencia social y religiosa, así como en la valoración de las tradiciones y diversos ritos paganos. Es una sociedad de fuertes contrastes donde las clases sociales están muy marcadas, tal y como indica el personal de la embajada.

La mujer juega un papel significativo en la sociedad y también en el mundo de los negocios, estando equiparado al del hombre.

La consideración del tiempo que tienen los brasileños se aproxima a la afirmación de time is eternal, por lo que la puntualidad no se practica en demasía, a pesar de lo cual, es importante establecer citas con bastante antelación.

La gastronomía es muy importante en el país, con mucha variedad.

En las comidas y cenas informales se puede hablar sobre negocios.

Son una sociedad de contacto, más recatado en el ámbito profesional, en el que suelen apostar por el uso del tratamiento formal de você y utilizar los títulos profesionales y académicos.

Respecto del idioma, el oficial es el portugués, pero suelen ser conocedores de español e inglés. Al igual que ocurre en otros países de América latina, muchos brasileños identifican español con gallego y desconocen, en líneas generales, el idioma gallego, pero suelen valorar su uso en un intento de aproximación lingüística y social.

Es aconsejable comenzar la relación con una charla informal, con preferencia por los temas deportivos y viajes; los referentes a la política, las desigualdades sociales o la corrupción son tratados en ambientes de más confianza. 
En las negociaciones valoran el factor emocional sobre lo racional, dando gran importancia a las tarjetas profesionales, que deben entregarse al inicio de la reunión y agradecerse debidamente cuando se reciben.

\subsection{Angola}

Angola tiene una sociedad reposada, paciente y alegre. Con un carácter afable, suelen mezclar el mundo profesional y familiar. Gesticulan mucho y se expresan usando un tono de voz bastante elevado.

Los angoleños sienten un profundo respeto por sus creencias y tradiciones y el peso de la religión y ritos ostentan un peso considerable en los negocios.

El valor del tiempo para los angoleños es bastante laxo, ya que son pausados en las conversaciones. La puntualidad no es muy practicada.

Se trata de una sociedad en la que el estatus social impera en el ámbito personal y profesional, por lo que se utilizan mucho los títulos profesionales y académicos. Las jerarquías están muy marcadas.

En cuanto al papel de la mujer en el ámbito de la negociación, cabe destacar que no está equiparado al del hombre, pero está mejorando.

En una primera toma de contacto deben utilizarse los tratamientos formales, de usted, y dirigirse a los angoleños haciéndolo por el nombre precedido de Sr. Sra. y/el tratamiento académico/profesional correspondiente. A partir de este momento inicial, cuando ya exista cierta confianza, se pasará a un tratamiento más coloquial.

Gustan de una conversación inicial distendida, en la que se deben evitar temas como las guerras o la colonización.

A pesar de que el portugués es la lengua más extendida, también es habitual en los negocios el uso del inglés. Suelen desconocer el gallego y su proximidad al portugués, por lo que no tienen formada opinión al respecto.

En cuanto a la gastronomía, cabe destacar sus influencias portuguesas y un alto consumo de pescado, carne y verduras. La bebida más habitual es la cerveza, pero está a empezando a imponerse el consumo de vino. En el ámbito de los negocios prefieren el whisky y, a la hora de brindar, optan por el champán.

Las reuniones de negocios suelen trasladarse a la mesa, y no les importa mezclar temas de conversación profesionales y de otra índole. 
En la negociación dan más importancia al factor emocional que al racional. Valoran mucho que la vista esté fija en el interlocutor, trasmitiendo serenidad y honestidad. Prefieren propuestas sencillas y bien presentadas.

Durante las negociaciones suelen servir cafés o aperitivos.

Las tarjetas de presentación son importantes, debiéndose entregar al inicio y recibiéndolas con agradecimiento. Respecto a los regalos, no es aconsejable entregarlos, a no ser que sean pequeños artículos de escaso valor económico, para evitar confusiones con sobornos. Las invitaciones en el ámbito de los negocios también son habituales, especialmente para ir a almorzar, actividades culturales o deportivas.

\subsection{Cabo Verde}

Los caboverdianos son persistentes y tenaces. Valoran mucho las tradiciones y las supersticiones. Mezclan vida profesional y personal, pero manifiestan distintas distancias físicas en un ambiente y otro.

Emplean el tratamiento de usted en las relaciones profesionales, así como los títulos profesionales. En este ámbito valoran también las jerarquías, que se hacen especialmente evidentes en los primeros encuentros.

Respeto del papel que juega la mujer en el ámbito de los negocios, aún es muy reducido.

Valoran el tiempo con parsimonia y para ellos el tiempo es "eterno", por lo que la puntualidad no se practica en exceso, a pesar de lo cual es recomendable respetar las citas de trabajo y fijarlas con una semana de antelación.

La gastronomía es muy valorada y también tiene cabida en el mundo de los negocios, de forma que en las comidas informales es acertado hablar de este tipo de asuntos. Suelen brindar para celebrar un éxito.

La mirada es muy importante y, al contrario que en la sociedad gallega, los silencios transmiten muchas emociones.

Se comunican en portugués y en criollo, lengua natal de la isla. Valoran los esfuerzos de los extranjeros por utilizar sus lenguas y, al igual que en Angola, desconocen el idioma gallego.

En las negociaciones hacen especial hincapié en los aspectos económicos y gustan de presentaciones sencillas. Las tarjetas de presentación son un recurso importante y deben entregarse al inicio del encuentro. Gustan 
de regalos de empresa y, de hecho, son habituales. No acostumbran a hacer ningún tipo de invitación, pero sí agradecen ser invitados.

\subsection{Portugal}

La sociedad portuguesa se caracteriza por la paciencia, la constancia, el respeto y la amabilidad como rasgos generales. Valoran las tradiciones y afirman ser una nación moderna y, en ese aspecto, abogan por un trato igualitario.

En un primer encuentro son muy formalistas y valoran la cortesía y formalidad. Para saludar, toman de base el apretón de manos, pero entre personas más allegadas utilizan el beso social y el abrazo. Se dirigen a las personas con la formalidad correspondiente, utilizando Sr., D. y los títulos académicos y/o profesionales.

Desde el punto de vista del protocolo institucional, cuentan con un orden de precedencias muy similar al español.

Es una sociedad de contacto que gusta comenzar con una charla distendida para romper el hielo, pero sin prolongarla en demasía.

Respeto de las costumbres y tradiciones, destacaremos que la invitación a casas particulares sólo se produce cuando ya existe una relación personal, no siendo necesario llevar un obsequio. En cuanto a los regalos, no son apropiados para un primer encuentro.

Para los portugueses, el tiempo se considera un bien preciado, por lo menos en lo que se refiere al trabajo. A pesar de esta máxima, los portugueses toman el tiempo necesario para afianzar la relación y pensar sobre las cuestiones que hay que tratar, por eso se estila un parloteo previo.

Dada la cercanía cultural e idiomática entre Galicia y Portugal, son muchos los portugueses que consideran que pueden desarrollar una conversación básica con cualquier gallego utilizando este idioma, pero a la hora de profundizar y tratar temas más complejos y específicos prefieren mantener la negociación en portugués o español y evitar el conocido "portuñol". Cabe destacar también que los portugueses cuentan con un alto grado de conocimiento de otras lenguas, especialmente el inglés, por lo que -en muchos casos- las negociaciones también se desarrollan en esta lengua. 
Dentro de la cultura y las tradiciones portuguesas destaca la gastronomía. Son habituales las comidas de negocios, aunque no para hablar de temas empresariales.

A la hora de la negociación, los portugueses esperan que sus interlocutores sean educados, formales y que aporten propuestas concretas donde prime lo racional sobre lo emocional. Las tarjetas profesionales son un elemento importante que se entrega al comienzo y se recibe con agradecimiento. Con respecto a las invitaciones, los portugueses se ciñen a invitar a reuniones formales y comidas de negocios; no realizan invitaciones en las que mezclen vida personal y profesional.

\subsection{Timor Oriental}

Timor Oriental cuenta con una sociedad paciente ante la adversidad. Son personas alegres y solidarias para los que la religión es uno de los pilares clave de la comunidad. Valoran la honestidad.

Las tradiciones y supersticiones consiguen un grado notable de importancia tanto en la vida diaria como en el mundo de los negocios. El estatus social es muy importante y está presente en todos los campos de la vida, pero especialmente en el ámbito de los negocios, donde a las reuniones acuden los máximos representantes del organismos y tienen toda la responsabilidad a la hora de negociar.

La mujer timorense no ostenta un papel importante en los negocios y su papel queda prácticamente reducido al de madre.

Los timorenses son una sociedad que trabaja para vivir e interpretan el tiempo en la concepción de time is money, por lo que consideran la puntualidad un factor fundamental. Los horarios de trabajo acostumbran a ser flexibles y las reuniones, que deben fijarse con una semana de antelación por lo menos, raramente se respetan para las fechas fijadas.

La gastronomía es muy importante y rica en variedad. No suelen brindar y las comidas de negocios tampoco son frecuentes.

Son una sociedad de contacto, aunque no les gusta ver invadido su espacio personal.

No gesticulan mucho y usan un tono de voz moderado. La mirada debe estar fija en el interlocutor. 
Hablan portugués y tetun y, cuando en el mundo de los negocios no es posible utilizarlos, acuden al inglés, que también es el idioma más habitual en la plasmación escrita de los documentos de ámbito internacional. Son muy formales y acostumbran a emplear el tratamiento de usted y dirigirse al interlocutor mediante la fórmula: título+nombre+apellido, además de los correspondientes títulos profesionales.

En la negociación prefieren presentaciones detalladas pero sencillas; valoran las tarjetas profesionales, que deben estar en portugués e inglés por lo menos, y se intercambian en el primer momento. Deben recibirse con agradecimiento y leerse antes de guardarlas. También agradecen los regalos empresariales, que se entregarán en el primer encuentro. Por el contrario, las invitaciones fuera del ámbito estrictamente empresarial no son habituales.

\section{Conclusiones}

Como principales conclusiones de este estudio, destacamos:

- La globalización es un fenómeno complejo que hace patente la necesidad de que un país establezca relaciones con otro, pues en la actualidad ninguna nación puede considerarse autosuficiente (O1, O2).

- El éxito de la economía y del tejido empresarial gallego radica en la internacionalización de su actividad, en su grado de inmersión de los nuevos mercados emergentes, como Brasil y Angola, y en el aprovechamiento de su idioma como ventaja competitiva en la lusofonía. Así lo entienden las organizaciones públicas gallegas, que han materializado en los últimos años diversas campañas promocionales dirigidas a los empresarios para convencerlos de que el uso del gallego puede ser útil y beneficioso a la hora de entablar negociaciones internacionales (O4).

- En el marco de la negociación internacional, Galicia y su tejido empresarial cuentan con una gran ventaja competitiva a la hora de entablar relaciones profesionales: el idioma.

La cercanía del gallego y el portugués guardan importantes similitudes lingüísticas, por lo que esta herramienta puede resultar crucial a la hora de entablar relaciones. Sin embargo, y a la vista de las entrevistas realizadas, tanto a personal de las embajadas como a empresarios gallegos que trabajan en esos 
países, es posible que la lengua gallega sirva como medio para comunicarse en la lusofonía y emprender negocios de talla internacional, a pesar de lo cual, para negociar en este ámbito empleando la lengua gallega como lengua de negocios es necesario dibujar un perfil detallado y específico de la sociedad y de la cultura lusófonas y de cada país o región en particular.

Los propios interlocutores reconocen que el ideal en una conversación entre empresarios gallegos y lusófonos es respetar ambas lenguas y hablar siempre de forma correcta (y despacio) su lengua materna para no mezclar palabras y, de esa forma, evitar ruidos comunicativos, como puede ser el conocido "portuñol" (O4).

- Por eso, los gallegos que deseen trabajar en el entorno lusófono deben estudiar en profundidad los patrones culturales de cada región y, por lo tanto, afianzar sus conocimientos en materia de comunicación no verbal, incluyendo la proxémica, kinesia o la cronémica, por lo que el interlocutor gallego debe esforzarse en informarse sobre los distintos valores que se le da al tiempo entre ambas culturas, la vestimenta, el saludo, la normas de comportamiento, las leyes de carácter protocolario, la gastronomía, la gestualidad, el papel de la mujer en el mundo de los negocios y la importancia y valor en cada cultura a la religión $(\mathrm{O} 4, \mathrm{O} 5)$.

- El espacio cultural lusófono es complejo y variado, dado que los países que lo componen se sitúan en puntos geográficos muy distanciados entre sí. No obstante, a grandes rasgos podemos afirmar que la sociedad lusófona es una sociedad de contacto, que se caracteriza por tener una personalidad respetuosa, tranquila y formal y que hoy en día los factores más influentes a la hora de establecer contactos de negocios son la religión, la cultura y la economía, que las tradiciones y el pasado histórico repercuten en el día a día y que el tiempo se considera un bien relativo $(\mathrm{O} 3, \mathrm{O} 5)$.

- En las negociaciones en el mundo lusófono, el factor racional tiene más peso que el emocional. La propuesta que se presenta debe ser sencilla, y una de las principales barreras a la hora de negociar es que el negociador extranjero desconozca la idiosincrasia del país. Además de eso, las conversaciones de negocios deben transcurrir 
con lentitud y el negociador extranjero debe presentar honestidad, seriedad, educación, imagen pulcra y capacidades para desarrollarse en otros países (O3, O5).

- El gallego es una lengua desconocida para el espacio lusófono -salvo en su entorno geográfico más próximo: Portugal-y para sus integrantes no es una opción tan válida como el portugués para negociar en el campo internacional. Sin embargo, aseguran que, gracias a las similitudes lingüísticas entre ambas lenguas, serían capaces de entender a un empresario que les hablase gallego y considerarían el gesto como positivo, pues se traduce en un esfuerzo por parte del negociador gallego de acercarse a su cultura.

En definitiva, no cabe duda de que el empresariado gallego debería abrirse camino en la lusofonía, buscar oportunidades de negocio en los mercados emergentes propuestos, apoyarse en su lengua como medio de comunicación, y salvar las diferencias culturales que presenta ese contexto aplicando los consejos recogidos a través de la experiencia personal de aquellos que viven inmersos en el espacio lusófono.

Llegados a este punto, podemos confirmar el supuesto de partida de esta investigación que indicaba que la cercanía lingüística entre el gallego y el portugués es evidente y va más allá de la mera concepción idiomática, llegando a ámbitos como el sociocultural, el político y el económico; sin embargo, esta cercanía no es total y existen limitaciones para el entendimiento mutuo que deben tenerse en cuenta a la hora de buscar relaciones internacionales exitosas, normalmente enmarcadas dentro del ámbito de la comunicación no verbal. Así queda de manifiesto a través del trabajo de campo, donde todos los entrevistados coindicen en la importancia de tener en cuenta no sólo los aspectos idiomáticos, sino también los relacionados con la comunicación no verbal y cultural.

\section{Referencias bibliográfícas}

AguAYo ARRABAL, Natividad. (1989) "El traductor-intérprete en el comercio exterior: ¿realidad o necesidad.” Revista Entreculturas 5, pp 57-74.

AGUIRRE BAZTÁN, Ángel. (1997) Cultura e identidad cultural. Barcelona: Bardenas. 
Alburquerque, Alexandra \& José Paulo Esperança (2010) El valor económico del portugués Lengua de conocimiento con influencia global. ARI (Real Instituto Elcano de Estudios Internacionales y Estratégicos), 127. Versión electrónica: <http://www.realinstitutoelcano.org/wps/wcm/connect/ cdca050043c57fa6931dff9a3710b050/ARI127-2010_Alburquerque_ Esperanca_valor_economico_portugues_lengua_influencia_global. pdf?MOD=AJPERES\&CACHEID=cdca050043c57fa6931dff9a3710b050> Álvarez, Rosario. (2009) "Retruque ao relatorio de Ivo Castro", en H. Monteagudo (Coord.), Sociedades plurilingües: da identidade á diversidade, (pp. 253-263). Santiago de Compostela: Consello da Cultura Galega. Versión electrónica: $<$ http://consellodacultura.org/mediateca/extras/sociedades_plurilingues.pdf> Antero Reto, Luis. (Coord.) (2012) Potencial Económico da Língua Portuguesa. Lisboa: Texto Editores.

BAGNO, Marcos. (2011) "O portugués não procede do latim. Uma proposta de classificação das línguasderivadas do galego." Grial: revista galega de cultura 191, pp. 34-39.

BILBENY, Norbert. (2005) Uno mismo y los otros. De las experiencias existenciales a la interculturalidad. Barcelona: Herder.

BioermatzKi, William E. (1986) "Intercultural communication." Communication Research Trends 7:3.

Carbaugh, Robert J. (2009) Economía Internacional. México D.F.: Cengage Learning Editores.

CAStro, Ivo. (2009) "Galicia no espazo cultural e simbólico da lusofonía." En: Monteagudo, Henrique (coord.) 2009. Sociedades plurilingües: da identidade á diversidade, (pp. 219-253). Versión electrónica: <http://consellodacultura. org/mediateca/extras/sociedades_plurilingues.pdf>

CORDAL, Constantino. (2012) "Eurorrexión, crise e Lusofonía".Informe anual sobre a acción exterior de Galicia: ¿Menos acción exterior para internacionalizarse máis?, pp. 21-23. Versión electrónica: <http://www.igadi.org/arquivo/ pdf/igadi_weintheworld_2011.pdf>

CUADRADO, Carmen. (2007) Protocolo y comunicación en la empresa y los negocios. Madrid: FC, Editorial.

DA Silva, Helena, Froilán, Pedro \& Carlos María Fernández (2009) La influencia del capital intelectual en la capacidad de innovación de las empresas del sector de automoción de la eurorregión Galicia Norte de Portugal. Cátedra Eurorrexión Galicia-Norte de Portugal, Universidade de Vigo. 
DANIELS, John, Radebaugh Lee. \& Daniel Sullivan (2004) Negocios internacionales: ambientes y operaciones (10 edición). México: Pearson Educación.

FERnÁNDEZ-JARdón FERnÁNDEZ, Carlos. (2008) Capital intelectual e internacionalización de la pyme gallega. Vigo: Universidade, Servizo de Publicacións.

FernÁndez Souto, Ana Belén; Vázquez Gestal, Montserrat \& Adriana Prada Iglesias (2016) "Más allá de la proximidad lingüística: Galicia y Portugal en la negociación internacional". Revista Contratexto, $\mathrm{n}^{\circ} 16$.

FERNÁNDEZ SOUTO, Ana Belén. (2016) Guía de protocolo y usos sociales. Madrid: Síntesis.

Gallego Hernández, Daniel; Geoffrey S., Koby \& Verónica Román Mínguez (2016). "Traducción económica, financiera y comercial: aproximación a aspectos teóricos. Estudio basado en encuestas."

García CAStaño, Francisco Javier \& Rafael Pulido Moyan (1992) "Educación Multicultural y Antropología de la Educación". En: Fermoso, Paciano (ed.) Educación Intercultural: la Europa sin fronteras. Barcelona: Narcea, pp. 35-69. GONZÁLEZ GURRIARÁN, Jorge et al. (2000) La internacionalización de la pequeña y mediana empresa gallega: Análisis, diagnóstico y posibles estrategias. Vigo: Consorcio Zona Franca.

GUdYKUnST, William. (1987) "Cross-Cultural Comparisons". En: Berger, Charles

$\&$ Steven Chafee (eds.): Handbook of Communication Science. London: Sage. Gudykunst, William \& Stella Ting-Toomey (1988) Culture and Interpersonal Communication. London: Sage.

JANDT, Fred. (1998) Intercultural Communication. An Introduction. California: SAGE Publications.

Ledo-Andión, Margarita, CAmpos, Francisco, Toural, Carlos, Rúas, Xosé, Rodríguez, Ana Isabel, Costa, Carmen, Salgueiro, María \& Marta RODRÍGUEZ (eds.) (2017) "Alén das fronteiras: Redes na diversidade." Actas del I Congreso Internacional de la Asociación Galega de Investigadores e Investigadoras en Comunicación, Universidade de Santiago de Compostela. LEDO-ANDIÓN, Margarita. (dir.) (2011) Lusofonía, Interactividade e interculturalidade, Servizo de Publicacións da Universidade de Santiago de Compostela Llamazares García-LOMAS, Olegario. (2011) "La influencia de la cultura en los negocios internacionales." Boletín económico de ICE, 3011, pp. 47-52 Versión electrónica: http://www.revistasice.com/CachePDF/BICE_3011_4752_DC1F87CE5EEC51F07F5B7E538EFE5D29.pdf. 
LÓPEZ FACAL, Xan. (2012) "Galiza e o desafío da internacionalización. We in theworld=Nos no Mundo." Informe anual sobre a acción exterior de Galicia 2011. ¿Menos acción exterior para internacionalizarse máis? Versión electrónica: <http://www.igadi.org/arquivo/pdf/igadi_weintheworld_2011.pdf>

MALDONADO, Karen Dayana. (2006) Aproximación a un marco conceptual y teórico sobre la interculturalidad de los negocios internacionales. Bogotá D.C.: Editorial Universidad del Rosario. Versión electrónica: <http://repository.urosario.edu. co/bitstream/10336/1208/1/BI\%2033.pdf>

Melo Alves, Fernanda María. (2007) Articulación y complementariedad de las políticas de la lengua portuguesa, de cooperación y de información en los países lusófonos: guía metodológica para la implantación de la Biblioteca Digital Lusófona (BDL). (Tesis Doctoral inédita), Universidad Carlos III. Madrid. Versión electrónica: <http://e-archivo.uc3m.es/handle/10016/2540>

Monteagudo Henrique \& Serafín Alonso Pintos (2010) "Que lusofonía para Galicia?" Grial: revista galega de cultura 193, pp. 77-85.

Oliveras, Àngels. (2000) Hacia la competencia intercultural en el aprendizaje de una lengua extranjera. Estudio del choque cultural y los malentendidos. Madrid: Edinumen, Memorias para el aprendizaje, Serie Máster E/LE Universidad de Barcelona.

Otero Simón, Miguel A. (2008) Internacionalización. Cómo iniciar la expansión de su empresa en el mercado internacional. A Coruña: Netbiblo.

REDACCIÓN. (2010) O Xornal de Galicia, 23/05/10

REDACCión. (2013) Galicia confidencial, 17/05/2013

REIS, Carlos. (2010) "La internacionalización de la lengua portuguesa". En AAVV, Ciclo de Conferencias 2010: El espacio ibérico de las lenguas. Instituto Cervantes, Madrid, 25 de marzo 2010. Versión electrónica: <http://www. cervantes.es/imagenes/File/lengua/jornadas/Carlos_Reis_2010IC.pdf>

Rial, Antonio, Valcarce, Margarita., Barreira, Eva et al. (2012) "Mobilidade, formación, orientación e emprego no ámbito transfronteirizo." Actas do XIII Congreso Internacional de Galicia e Norte de Portugal de Formación para o Traballo, Servizo de Publicacións da Universidade de Santiago de Compostela. Rodrigo Alsina, Miquel. (1999) Comunicación intercultural. Barcelona: Anthropos, Colección Autores, Textos y Temas Ciencias Sociales, n. 22.

Rodrigo Alsina, Miquel. (2007) "Impacto de la cultura en los negocios internacionales, un énfasis en los negocios con México". Daena: International 
Journal of Good Conscience 2 (2), pp. 156-228. Versión electrónica: <http:// www.spentamexico.org/v2-n2/2(2)\%20156-228.pdf>

Rugman, Alan \& Richard Hodgetts (1997) Negocios internacionales. Un enfoque de administración estratégica. México: McGraw-Hill.

SÁEz PONTE, Enrique. (2012) "Apuntes para unha estratexia exterior cara os países emerxentes". En AAVV, Informe anual sobre a acción exterior de Galicia: ¿Menos acción exterior para internacionalizarse máis?, pp.17-19. Versión electrónica: <http://www.igadi.org/arquivo/pdf/igadi_weintheworld_2011.pdf> Sosoni, Vilelmini \& Łucja Biel. (2018) "EU Legal Culture and Translation." International Journal of Language \& Law (JLL) 7.

TRILlo, Juan Manuel \& Rubén C. LoIS, Rubén (2011) "La frontera como motivo de atracción: una breve mirada a las relaciones Galicia-Regiao Norte", Geopolítica(s) 2:1, pp. 109-134.

VAQUEIRO, Iván. (Asociación Galega da Língua). (2013) <www.pglingua.org/ noticias/entrevistas/5942-ivan-vaqueiro-diretor-de-projetos-dela-fegapelsente-si-claro-la-falta-de-nao-tener-mecanismos-institucionales-que-nospermitam-acercar-nos-a los-paises-lusofonos>

VARIOS AUTORES. (A Mesa pola Normalización Lingüística). (2009) Que facemos. Actividades. Recuperado de: <http://www.amesanl.org/gl/mesa/actividades>

VARIOS AUTORES (Asociación Galega da Língua). (2010) AGAL. As nossas actividades. Versión electrónica: <http://www.agal-gz.org/corporativo/index. php?option=com_content $\&$ view=article $\&$ id $=88 \&$ Itemid $=12>$

VARIOS AUTORES. (Academia Galega da Língua Portuguesa). Versión electrónica: $<$ http://academiagalega.org/normasde-regime-interno/normas-de-regimeinterno-da-academia-galega-da-lingua-portuguesa.html>

WatzlaWick, Paul; Janet Beavin \& Don Jackson (1971) Teoría de la comunicación humana. Buenos Aires: Tiempo Contemporáneo.

\section{BIONOTAS / BIONOTES}

Ana BelÉn Fernández Souto, Profesora Titular de Universidad en la Facultade de Ciencias Sociais y de la Comunicación de la Universidade de Vigo (España). Especializada en comunicación organizacional, relaciones públicas y protocolo internacional. Cuenta con 2 sexenios de investigación. 
ANA BelÉn FernÁndez Souto, Professor in the Facultade of Sciences Sociais and of the Communication of the Universidade of Vigo (Spain). Specialized on organizational communication, public relations and international protocol. She has recognised 2 research six-year periods.

José RÚas ARAújo, Profesor Titular de Universidad en la Facultade de Ciencias Sociais y de la Comunicación de la Universidade de Vigo (España). Especializada en comunicación política. Cuenta con 1 sexenio de investigación.

JosÉ RÚAS ARAÚJO, Professor in the Facultade of Sciences Sociais and of the Communication of the Universidade of Vigo (Spain). Specialized on political communication. He has recognised 1 research six-year period.

Adriana Prada IGLESIAS, Graduada en la Facultade de Ciencias Sociais y de la Comunicación de la Universidade de Vigo (España)

Adriana Prada IGLESIAS, graduated in the Facultade of Sciences Sociais and of the Communication of the Universidade of Vigo (Spain) 